\title{
Human Papillomavirus-6
}

National Cancer Institute

\section{Source}

National Cancer Institute. Human Papillomavirus-6. NCI Thesaurus. Code C14319.

Genital warts caused by HPV 6 and 11 are the most common STD in the United States.

Over a million cases are seen per year. 